Published in final edited form as:

Psychiatr Serv. 2011 April ; 62(4): 404-410. doi:10.1176/appi.ps.62.4.404.

\title{
Effects of Sexual Orientation and Gender on Perceived Need for Treatment by Persons With and Without Mental Disorders
}

\author{
Christine E. Grella, Ph.D. ${ }^{a}$, Susan D. Cochran, Ph.D., M.S. ${ }^{c, d}$, Lisa Greenwell, Ph.D. ${ }^{a}$, and \\ Vickie M. Mays, Ph.D., M.S.P.H. b,c \\ alntegrated Substance Abuse Programs, Semel Institute for Neuroscience and Human Behavior, \\ UCLA, Los Angeles, CA \\ bDepartment of Psychology and Department of Health Services, School of Public Health, UCLA, \\ Los Angeles, CA \\ 'Center for Research, Education, Training and Strategic Communications on Minority Health \\ Disparities, UCLA, Los Angeles, CA \\ dDepartment of Epidemiology, School of Public Health and Department of Statistics, UCLA, Los \\ Angeles, CA
}

\begin{abstract}
Objective-Research has shown that some individuals with sexual minority status have a higher prevalence of psychiatric disorders (both mental health and substance use) and also tend to seek treatment at higher rates as compared with similar heterosexuals. Relationships among treatment need and treatment use, however, are not well understood. This paper examines the relationship of sexual orientation and gender to perceptions of need and unmet need for treatment among individuals with and without mental health or substance use disorders.

Methods-Data were obtained from a probability sample of California residents that oversampled for sexual minorities (unweighted $\mathrm{N}=2,079$ ). Bivariate analyses compared perceived treatment need and treatment use among groups defined by sexual orientation, gender, and presence of a mood, anxiety, or substance use disorder. Logistic regression models were fit predicting absence of treatment among those who perceived a need for it (unmet need), testing the interactive effects of gender, disorder, and sexual orientation and controlling for sociodemographics.
\end{abstract}

Results-Sexual minority women had about half the odds of unmet need for treatment compared with other women, but there was no interaction between sexual minority status and unmet treatment need for men. Among individuals without any of the disorders measured, sexual minorities had lower odds of unmet need for treatment compared with others. Heterosexual men and women were most likely to have unmet treatment needs.

Conclusions-Sexual orientation and gender differentially influence treatment utilization, particularly among those who do not have a diagnosed disorder but perceive a need for treatment. Diagnostic criteria appear to be less relevant to understanding treatment use among sexual minorities.

\section{Keywords}

mental health disorders; substance use disorders; sexual minority orientation; need for treatment; unmet treatment need 


\section{Introduction}

Epidemiological studies have shown higher prevalences of alcohol and drug disorders in some sexual minority populations (1-7). Similarly, the prevalence of some commonly occurring psychiatric disorders appears to be elevated within sexual minorities $(3,8,9)$. However, it is not clear whether sexual minorities also have higher rates of treatment seeking for these disorders, as compared with heterosexuals. Prior research has shown that lesbian and bisexual women have higher rates of help-seeking for alcohol problems, even among those with lower levels of alcohol-related problems $(4,10,11)$. Similarly, studies have shown that lesbians tend to utilize mental health services at higher rates as compared with heterosexual women (12).

Cochran and Mays (13) found that men and women who reported same-gender sex partners were more likely than their respective counterparts to have sought mental health/substance abuse services in the past year. However, a study of gay men in Geneva showed that nearly half of those with a psychiatric disorder (including substance use) had never sought treatment, comparable to rates found in other general population studies (14). In contrast, studies with treatment samples have shown more severe substance use problems and higher rates of psychiatric treatment among sexual minorities compared with heterosexuals in substance abuse treatment (15). But clinical studies with targeted sub-groups, such as gay men who are methamphetamine users, may show elevated levels of psychopathology as compared with population-based samples of sexual minorities $(16,17)$.

Moreover, there is disagreement about the factors that prompt individuals to seek treatment for psychiatric disorders (18). Several national surveys have found a lack of concordance between the presence of a psychiatric disorder and the receipt of treatment services $(19,20)$. Most individuals with these disorders neither receive treatment for them nor perceive a need for it $(21,22)$. Individuals most commonly state that they can manage these problems on their own (23) and/or are "not ready" to stop using substances (24). National epidemiological surveys demonstrate that some individuals who do not have a diagnosable disorder seek treatment (25) or perceive a need for it (26), especially when they have high levels of psychological distress or impairments in functioning (27-29).

\section{Current paper}

The goal of the present paper is to examine the effects of sexual orientation on perceived need and unmet need for treatment of mental health and substance use disorders, taking into consideration whether this relationship is differentially influenced by gender as well as the presence of a diagnosed disorder. Given prior research showing more treatment seeking among sexual minorities, and higher rates of health services utilization among women (30), we hypothesize that sexual minorities, particularly women, will be more likely than heterosexuals both to perceive a need for and to receive treatment, yielding overall lower levels of unmet need for treatment, after controlling for other socio-demographic characteristics that may be independently associated with treatment access and utilization.

\section{Methods}

\section{Study design}

Data for this study come from the 2004-2005 California Quality of Life Survey (Cal-QOL) (31) which is a follow-back to the 2003 California Health Interview Survey (CHIS). The parent CHIS is a stratified multistage random-digit telephone health surveillance interview of more than 42,000 adults aged 18 years and older conducted every other year since 2001. Information collected covers a wide range of topics, including health status, health 
conditions, health-related behaviors, health insurance coverage, and access to and use of health care services.

The Cal-QOL follow-back survey used a subsample of the original CHIS survey sample to obtain more detailed information about specific topics (in this case, regarding sexual orientation). The overall CHIS response rate was 34\% (using the American Association for Public Opinion Research Response Rate 4 method), which is consistent with other recent random-digit telephone interviews. In the CHIS, all adult respondents aged 18 to 70 years were asked about the genders of their sexual partners during the past year, and those aged 18 and older (with no age limit) were asked about their sexual orientation identity. Seventy-six percent of respondents were willing to participate in additional health surveys. From the CHIS sample, 4,165 individuals were selected by probability methods. Eligibility was determined by having completed either a CHIS interview in either English or Spanish; a willingness to be recontacted; and an over-selection for sexual orientation minority status. Of these, 2,322 individuals were successfully interviewed between October 2004 and February 2005 (56\% response rate using the American Association for Public Opinion Research Response Rate 1 method). (See Cochran and Mays (31) and Narayanan et al. (32) for more in-depth information on California Quality of Life Survey methods.) This study received Institutional Review Board approval from UCLA and Westat (the survey subcontractor).

\section{Study sample}

The current study used data from 2,079 (unweighted) individuals who were aged 18 to 64 years at the time of the CHIS interview, excluding those who were likely eligible for Medicare. Overall, the weighted sample was approximately half male (48.5\%) and half female $(51.5 \%)$. The ethnic/racial distribution was approximately $55 \%$ white, $30 \%$ Hispanic, 6\% African American, and 9\% of other ethnic groups (i.e., Asian/Pacific Islander, Native American). The mean age was 40.7 years. Most (72\%) were currently employed and $87 \%$ had health insurance (including both public and private insurers). Approximately $64 \%$ had at least some college education and were currently married or cohabiting with a partner, and the remainder were either dating or single.

\section{Measures}

Treatment-related measures-Need for treatment was assessed by two questions asking: "In the past 12 months, did you think you needed help for alcohol or other drug problems" or for "emotional or mental health problems, such as feeling sad, blue, anxious, or nervous?" A positive response to either question was coded to indicate a perceived need for treatment.

Treatment received was assessed by a number of questions. The first asked whether the respondent had "received any treatment for emotional, mental health, alcohol or other drug problems" in the past 12 months. Subsequent questions referred to treatment received for either type of disorder in specific venues, including hospitalization; residential substance abuse treatment; emergency rooms; treatment from a mental health provider or substance abuse specialist (i.e., a psychiatrist, psychologist, social worker, psychiatric nurse, or counselor); self-help groups; alternative medicine; or a non-residential alcohol or other drug treatment program.

Unmet need for treatment was derived by coding those who perceived a need for treatment, but who did not receive any in the past 12 months, as "yes" and all others who perceived a need for treatment as "no." 


\section{Presence of disorder}

Diagnoses were derived using the Composite International Diagnostic Interview-Short Form (CIDI-SF) (33). The CIDI-SF, administered by trained lay interviewers, provides screening assessments of community samples for common mental disorders. Studies have found moderate agreement between CIDI-SF diagnoses and those obtained by face-to-face diagnostic clinical interviews $(34,35)$. Probable diagnoses were obtained for past-year major depression, generalized anxiety disorder, and panic attacks using $D S M-I I I-R$ criteria (36).

Two additional CIDI-SF modules assessed past-year symptoms of drug and alcohol dependency. All respondents who indicated any use of illicit drugs or non-prescribed medications in the previous 12 months were administered the drug module which assesses 7 symptoms from 6 of the core symptom areas of substance dependency: a) using in larger amounts or for longer periods of time than intended, b) being under the influence or recovering from use while engaged in social obligations, c) experiencing emotional or physical problems from use, d) having an irresistible urge to use, e) spending a great deal of time using or getting over using, and f) developing tolerance. Those evidencing 3 or more of the 7 symptoms received a positive diagnosis for past-year probable drug dependency disorder consistent with modified DSM-IV criteria (37). Seven similar questions were asked concerning alcohol use, with the addition of an item on alcohol-related withdrawal symptoms. A diagnosis of past-year probable alcohol dependency disorder was made if the respondent reported symptoms in 3 or more of the 7 core areas consistent with DSM-IV criteria. This diagnostic screening method was shown to have excellent reliability and validity in identifying individuals with substance use disorders in the National Comorbidity Survey (38).

For descriptive purposes, disorders were classified into two (non-mutually exclusive groups): mood or anxiety (21.7\% of the weighted sample) and alcohol or drug use disorder ( $7.1 \%$ of the weighted sample). A composite variable indicating presence of any disorder versus no disorder was used in the multivariate analyses described below.

\section{Sexual orientation}

Sexual orientation was determined by information obtained about both behavioral histories and self-identification. Behavioral questions asked about the genders of sexual partners since age 18 years and during the past year. Individuals were also asked their sexual orientation identity. Respondents were classified into one of four categories: lesbian, gay, bisexual, or homosexually experienced women (hereafter referred to as "lesbian/bisexual women"; $13.8 \%$ of the unweighted sample; $5.2 \%$ of weighted sample); gay, bisexual, or homosexually experienced men (hereafter referred to as "gay and bisexual men"; $15.3 \%$ unweighted; $7.0 \%$ weighted); exclusively heterosexual women (37.7\% unweighted; $46.2 \%$ weighted); and exclusively heterosexual men ( $33.1 \%$ unweighted; $41.6 \%$ weighted).

\section{Statistical analyses}

Data were analyzed with SAS version 9.1.3. We applied sample weighting to adjust for selection probability, nonresponse, and post-stratification to generate estimates representative of the California population. We first investigated rates of past-year treatment need and received, and unmet need by groups classified by gender, sexual orientation, and presence of disorder using Chi-square tests. When cell sizes were less than 5, Monte Carlo estimates of exact tests were used to assess their significance. Second, in order to test the study hypothesis on the relationship of gender, disorder, and sexual orientation status on treatment use, unmet need for treatment in the past year was modeled as a dependent variable in logistic regressions. Age, race/ethnicity (white vs. others), and education level (some college vs. less) were entered as covariates. Interaction effects were examined by 
using the specified levels approach, which can be used when the modifier has two levels (39). The specified levels approach omits the predictor from the model and includes the modifier and two product terms - that is, the product of the predictor with each level of the modifier. The coefficients estimated for these two product terms can then be interpreted as the effect of the predictor for each level of the modifier.

Odds ratios (OR) and 95\% Wald confidence intervals (CI) are presented. Statistical significance is determined at the $p<0.05$ level. Logistic regression models presented all had acceptable fit, as indicated by the Hosmer-Lemeshow Goodness-of-Fit statistic (40).

\section{Results \\ Differences among sexual orientation groups}

Sexual minorities reported higher rates of mental health and substance use disorders (see Table 1). Lesbian/bisexual women had the highest prevalence of mood/anxiety disorders $(38.1 \%)$, followed by gay and bisexual males, heterosexual females, and heterosexual males. Gay and bisexual males had the highest prevalence of substance use disorders (17.7\%), followed by lesbian/bisexual women, heterosexual males, and heterosexual females.

With regard to socio-demographic characteristics, there were significant differences among the groups on age, race/ethnicity, education, employment status, and marital/cohabitation status. The sexual minority groups had higher proportions of whites and lower proportions of Hispanics and those of "other" ethnic groups, higher levels of education, and lower rates of marriage or cohabitation. Gay and bisexual males were also significantly older than heterosexual males. Regardless of sexual orientation, a greater proportion of men than women were employed in the past year.

\section{Treatment status by disorder, gender, and sexual orientation}

The three treatment-related outcomes were examined by gender, sexual minority, and disorder status (see Table 2). As expected, a greater proportion of those who had a mental health or substance use disorder perceived a need for treatment. Among those with at least one of these disorders, lesbian and bisexual women had the highest proportion reporting a need for treatment $(70.5 \%)$, followed by heterosexual women, gay and bisexual men, and heterosexual men. Similarly, among those without any disorder, lesbian/bisexual women had the highest proportion who reported a need for treatment (26.1\%), followed by gay and bisexual men, heterosexual women, and heterosexual men.

Overall, approximately $22 \%$ of the sample reported having received some form of treatment for a mental health and/or substance use problem in the past year. A higher proportion of those with disorders reported having received treatment compared to those without a disorder. Among those with and without disorders, lesbians and bisexual women had the highest rates of treatment received, whereas heterosexual men had the lowest.

About $41 \%$ of those expressing a need for treatment met the criterion for unmet need for treatment. Unmet need was highest among heterosexual men and women without disorders and lowest among lesbian/bisexual women regardless of disorder status. About $40 \%$ of gay and bisexual men regardless of disorder status had an unmet need for treatment.

\section{Logistic regression models of unmet need for treatment}

Unmet need for treatment was modeled in a logistic regression in which interactions among disorder status, gender, and sexual orientation were examined. All models were adjusted for the effects of age, race/ethnicity (white vs. Non-white), and education level. 
Using the specified levels approach, we examined the effect of sexual orientation within gender categories, and the effects of sexual orientation and gender within disorder categories. The results indicated that lesbian/bisexual women had about half the odds of unmet need for treatment compared to exclusively heterosexual women $(\mathrm{OR}=0.47, \mathrm{CI}$ : $0.22,0.99$; see Table $3,1^{\text {st }}$ column). However, there were no significant effects of sexual orientation for men on unmet need for treatment. Among those with no disorder, sexual minorities had about one third the odds of heterosexuals of unmet need for treatment $(\mathrm{OR}=$ 0.35 , CI: $0.15,0.85$; see Table $3,2^{\text {nd }}$ column). There was no significant interaction of gender and disorder status on unmet need for treatment (Table $3,3^{\text {rd }}$ column).

We also estimated this model without interaction terms in order to examine the main effects of gender, sexual orientation, and presence of a disorder (these data not shown). Findings indicated that men had higher odds than women of having unmet need (OR $=1.69, \mathrm{CI}: 1.10$, $2.58, \mathrm{p}<.05)$ and those with a disorder had lower odds of having unmet need than those without a disorder $(\mathrm{OR}=0.57, \mathrm{CI}: 0.37,0.87, \mathrm{p}<.05)$. However, there was no significant main effect of sexual orientation on unmet treatment need. Also, consistent with prior research (41), older individuals and whites had lower odds of having unmet need for treatment than their respective counterparts.

\section{Discussion}

This study extends prior research on the effects of sexual minority orientation on the prevalence of substance use and mental disorders to examine the relationship of prevalence with perceived need for and receipt of treatment services. Consistent with prior research, our findings indicated that sexual minorities had a greater prevalence of specific mental health and substance use disorders. Also, they had higher levels of perceived need for treatment and higher rates of treatment utilization, and therefore were the least likely to have unmet need for treatment. Yet when adjusted for socio-demographics, the main effect of sexual orientation on unmet need was no longer significant. The effect of sexual minority status was evident, however, in its interactions with gender and disorder status. Lesbian/bisexual women were significantly less likely than heterosexual women to have unmet treatment need, although there was no difference among men by sexual orientation. Sexual minority status was also significantly associated with unmet need for treatment among those without a diagnosed disorder, but was not among those with a disorder.

These findings suggest that sexual minority status may promote help seeking for psychiatric disorders $(42,43)$, but that its effect is moderated by gender. This finding is similar to a recent study using national survey data showing that the effects of sexual minority status on substance use and dependence were larger for women than men (44). However, in our study, the effect of sexual minority status was most apparent among women without a mental health or substance use disorder.

The findings from our population-based study suggest that diagnostic criteria may be less relevant to determination of perceived treatment need and actual utilization among sexual minority populations. This finding deserves greater exploration as to the factors that may propel sexual minorities to seek care (e.g., a greater value on therapeutic services, higher perceived stress that does not reach diagnostic thresholds), particularly in the face of the limited availability of services tailored for sexual minorities and the barriers to providing these services (45-47).

\section{Study limitations}

This study encountered limitations typical of telephone-based follow-back surveys. Although the Cal-QOL follow-back survey oversampled for sexual minorities, the weighted 
sizes of sexual orientation minority groups were relatively small. Statistical power may have been limited in determining significance for some relationships given the small sample sizes of some sub-groups. Although data were obtained for the most prevalent mental health disorders among population-based samples (48), namely mood and anxiety disorders, it is possible that those who were classified as "without disorders" had other mental health disorders that were not included in the Cal-QOL assessment, leading to their misclassification. Inclusion of variables on sexual orientation and sexual behavior in largerscale national probability mental health/substance use studies could offer important opportunities to investigate the robustness of our findings.

\section{Conclusion}

This study provides evidence that perceptions of need for treatment for either a mental health or substance use disorder are highly influenced by sexual orientation, and that treatment use is further influenced by both gender and sexual orientation. Moreover, the influence of gender and sexual orientation are more pronounced among those who do not meet diagnostic criteria for either type of disorder, but who nonetheless perceive a need for treatment. This suggests that the relationship of diagnostic criteria to determinations of treatment need among individuals with minority sexual orientation should be re-evaluated in order to better understand and more fully address the factors that influence treatment seeking among those who perceive a need for care.

\section{Acknowledgments}

Financial support for this work was obtained from the National Institute on Drug Abuse (DA 15539, DA 20826), and the National Center for Minority Health and Health Disparities (MD 000508). We also thank staff at the California Health Interview Survey for their assistance in making the Cal-QOL survey possible.

\section{Reference}

1. Burgard SA, Cochran SD, Mays VM. Alcohol and tobacco use patterns among heterosexually and homosexually experienced California women. Drug and Alcohol Dependence. 2005; 77:61-70. [PubMed: 15607842]

2. Cochran SD, Ackerman D, Mays VM, et al. Prevalence of non-medical drug use and dependence among homosexually active men and women in the US population. Addiction. 2004; 99:989-998. [PubMed: 15265096]

3. Gilman SE, Cochran SD, Mays VM, et al. Risk of psychiatric disorders among individuals reporting same-sex sexual partners in the National Comorbidity Survey. American Journal of Public Health. 2001; 91:933-939. [PubMed: 11392937]

4. Hughes TL, Hass AP, Razzano L, et al. Comparing lesbians' and heterosexual women's mental health: a multisite survey. Journal of Gay \& Lesbian Social Services. 2000; 11:57-76.

5. Scheer S, Peterson I, Page-Shafer K, et al. Sexual and drug use behavior among women who have sex with both women and men: results of a population-based survey. American Journal of Public Health. 2002; 92:1110-1112. [PubMed: 12084692]

6. Stall R, Paul JP, Greenwood G, et al. Alcohol use, drug use and alcohol-related problems among men who have sex with men: the Urban Men's Health Study. Addiction. 2001; 96:1589-1601. [PubMed: 11784456]

7. Wilsnack SC, Hughes TL, Johnson TP, et al. Drinking and drinking-related problems among heterosexual and sexual minority women. Journal of Studies on Alcohol and Drugs. 2008; 69:129139. [PubMed: 18080073]

8. Cochran SD, Mays VM, Sullivan JG. Prevalence of mental disorders, psychological distress, and mental health services use among lesbian, gay, and bisexual adults in the United States. Journal of Consulting and Clinical Psychology. 2003; 71:53-61. [PubMed: 12602425] 
9. Sandfort TG, de Graaf R, Bijl RV, et al. Same-sex sexual behavior and psychiatric disorders: findings from the Netherlands Mental Health Survey and Incidence Study (NEMESIS). Archives of General Psychiatry. 2001; 58:85-91. [PubMed: 11146762]

10. Drabble L, Midanik LT, Trocki K. Reports of alcohol consumption and alcohol-related problems among homosexual, bisexual and heterosexual respondents: results from the 2000 national alcohol survey. Journal of Studies on Alcohol. 2005; 66:111-120. [PubMed: 15830911]

11. Hughes TL. Lesbians' drinking patterns: beyond the data. Substance Use \& Misuse. 2003; 38:1739-1758. [PubMed: 14582576]

12. Bradford J, Ryan C, Rothblum ED. National Lesbian Health Care Survey: implications for mental health care. Journal of Consulting and Clinical Psychology. 1994; 62:228-242. [PubMed: 8201059]

13. Cochran SD, Mays VM. Relation between psychiatric syndromes and behaviorally defined sexual orientation in a sample of the US population. American Journal of Epidemiology. 2000; 151:516523. [PubMed: 10707921]

14. Wang J, Häusermann M, Ajdacic-Gross V, et al. High prevalence of mental disorders and comorbidity in the Geneva gay men's health study. Social Psychiatry and Psychiatric Epidemiology. 2007; 42:414-420. [PubMed: 17450453]

15. Cochran BN, Cauce AM. Characteristics of lesbian, gay, bisexual, and transgender individuals entering substance abuse treatment. Journal of Substance Abuse Treatment. 2006; 30:135-146. [PubMed: 16490677]

16. Peck JA, Shoptaw S, Rotheram-Fuller E, et al. HIV-associated medical, behavioral, and psychiatric characteristics of treatment-seeking, methamphetamine-dependent men who have sex with men. Journal of Addictive Diseases. 2005; 24:115-132. [PubMed: 16186088]

17. Peck JA, Reback CJ, Yang X, et al. Sustained reductions in drug use and depression symptoms from treatment for drug abuse in methamphetamine-dependent gay and bisexual men. Journal of Urban Health. 2005; 82:1100-108. [PubMed: 15738315]

18. Mechanic D. Is the prevalence of mental disorders a good measure of the need for services? Health Affairs. 2003; 22:8-20. [PubMed: 14515877]

19. Rabinowitz J, Gross R, Feldman D. Correlates of a perceived need for mental health assistance and differences between those who do and do not seek help. Social Psychiatry and Psychiatric Epidemiology. 1999; 34:141-146. [PubMed: 10327839]

20. Stockdale SE, Klap R, Belin TR, et al. Longitudinal patterns of alcohol, drug, and mental health need and care in a national sample of U.S. adults. Psychiatric Services. 2006; 57:93-99. [PubMed: 16399968]

21. Grella CE, Karno MP, Warda US, et al. Perceptions of need and help received for alcohol or drug dependence in a national probability survey. Psychiatric Services. 2009; 60:1068-1074. [PubMed: 19648194]

22. Wang PS, Lane M, Olfson M, et al. Twelve-month use of mental health services in the United States: results from the national comorbidity survey replication. Archives of General Psychiatry. 2005; 62:629-640. [PubMed: 15939840]

23. van Beljouw I, Verhaak P, Prins M, et al. Reasons and determinants for not receiving treatment for common mental disorders. Psychiatric Services. 2010; 61:250-257. [PubMed: 20194401]

24. National Survey on Drug Use and Health (NSDUH). Reasons for Not Receiving Substance Abuse Treatment. Substance Abuse and Mental Health Services Administration, Office of Applied Studies; Rockville, MD: 2003.

25. Kessler RC, Demier O, Frank RG, et al. Prevalence and treatment of mental disorders. New England Journal of Medicine. 2005; 352:2515-2523. [PubMed: 15958807]

26. Edlund MJ, Unützer J, Curran GM. Perceived need for alcohol, drug, and mental health treatment. Social Psychiatry and Psychiatric Epidemiology. 2006; 41:480-487. [PubMed: 16565918]

27. Druss BG, Wang PS, Sampson NA, et al. Understanding mental health treatment in persons without mental diagnoses: results from the national comorbidity survey replication. Archives of General Psychiatry. 2007; 64:1196-1203. [PubMed: 17909132] 
28. Narrow WE, Rae DS, Robins LN, et al. Revised prevalence estimates of mental disorders in the United States: using a clinical significance criterion to reconcile 2 surveys' estimates. Archives of General Psychiatry. 2002; 59:115-123. [PubMed: 11825131]

29. Regier DA, Kaelber CT, Rae DS, et al. Limitations of diagnostic criteria and assessment instruments for mental disorders: implications for research and policy. Archives of General Psychiatry. 1998; 55:109-115. [PubMed: 9477922]

30. Green CA, Popea CR. Gender, psychosocial factors and the use of medical services: a longitudinal analysis. Social Science \& Medicine. 1999; 48:1363-1372. [PubMed: 10369437]

31. Cochran SD, Mays VM. Physical health complaints among lesbians, gay men, and bisexual and homosexually experienced heterosexual individuals: results from the California Quality of Life Survey. American Journal of Public Health. 2007; 97:2048-2055. [PubMed: 17463371]

32. Narayanan, V.; Fry, S.; Fan, J. California Quality of Life Survey: methods Report. WESTAT; Rockville, MD: 2005.

33. Kessler RC, Andrews G, Mroczek D, et al. The World Health Organization Composite International Diagnostic Interview Short-Form (CIDI-SF). International Journal of Methods in Psychiatric Research. 1998; 7:171-185.

34. Aalto-Setala T, Haarasilta L, Marttunen M, et al. Major depressive episode among young adults: CIDI-SF versus SCAN consensus diagnoses. Psychological Medicine. 2002; 32:1309-1314. [PubMed: 12420900]

35. Talati A, Fyer AJ, Weissman MM. A comparison between screened NIMH and clinically interviewed control samples on neuroticism and extraversion. Molecular Psychiatry. 2008; 13:122-130. [PubMed: 17938631]

36. American Psychiatric Association. Diagnostic and Statistical Manual of Mental Disorders. 3rd ed revised (DSM-III-R). Author; Washington, DC: 1987.

37. American Psychiatric Association. Diagnostic and Statistical Manual of Mental Disorders. 4th ed (DSM-IV). Author; Washington, DC: 1994.

38. Epstein, JF.; Gfroerer, JC. Estimating substance abuse treatment need from a national household survey, analyses of substance abuse and treatment need issues. Office of Applied Studies, Substance Abuse and Mental Health Services Administration; Rockville, MD: 1995.

39. Van Ness, PH.; Allore, HG. Using SAS to investigate effect modification. Yale University School of Medicine; New Haven, CT: 2006. Available at http://www2.sas.com/proceedings/ sugi31/195-31.pdf

40. Hosmer, DW.; Lemeshow, S. Applied Logistic Regression. 2nd ed. John Wiley and Sons; New York: 2000.

41. Wells K, Klap R, Koike A, et al. Ethnic disparities in unmet need for alcoholism, drug abuse, and mental health care. American Journal of Psychiatry. 2001; 158:2027-2032. [PubMed: 11729020]

42. Andersen, RM. Behavioral Model of Families' Use of Health Services. University of Chicago, Center for Health Administration Studies; Chicago: 1968. Research series no. 25

43. Andersen RM. Revisiting the behavioral model and access to medical care: does it matter? Journal of Health and Social Behavior. 1995; 36:1-10. [PubMed: 7738325]

44. McCabe SE, Hughes TL, Bostwick WB, et al. Sexual orientation, substance use behaviors and substance dependence in the United States. Addiction. 2009; 104:1333-1345. [PubMed: 19438839]

45. Mayer KH, Bradford JB, Makadon HJ, et al. Sexual and gender minority health: what we know and what needs to be done. American Journal of Public Health. 2008; 98:989-995. [PubMed: 18445789]

46. Cochran BN, Peavy KM, Cauce AM. Substance abuse treatment providers' explicit and implicit attitudes regarding sexual minorities. Journal of Homosexuality. 2007; 53:181-207. [PubMed: 18032292]

47. Cochran BN, Peavy KM, Robohm JS. Do specialized services exist for LGBT individuals seeking treatment for substance misuse? A study of available treatment programs. Substance Use \& Misuse. 2007; 42:161-176. [PubMed: 17366131]

48. Grant BF, Stinson FS, Dawson DA, et al. Prevalence and co-occurrence of substance use disorders and independent mood and anxiety disorders: Results from the national epidemiologic survey on 
alcohol and related conditions. Archives of General Psychiatry. 2004; 61:807-816. [PubMed: 15289279] 


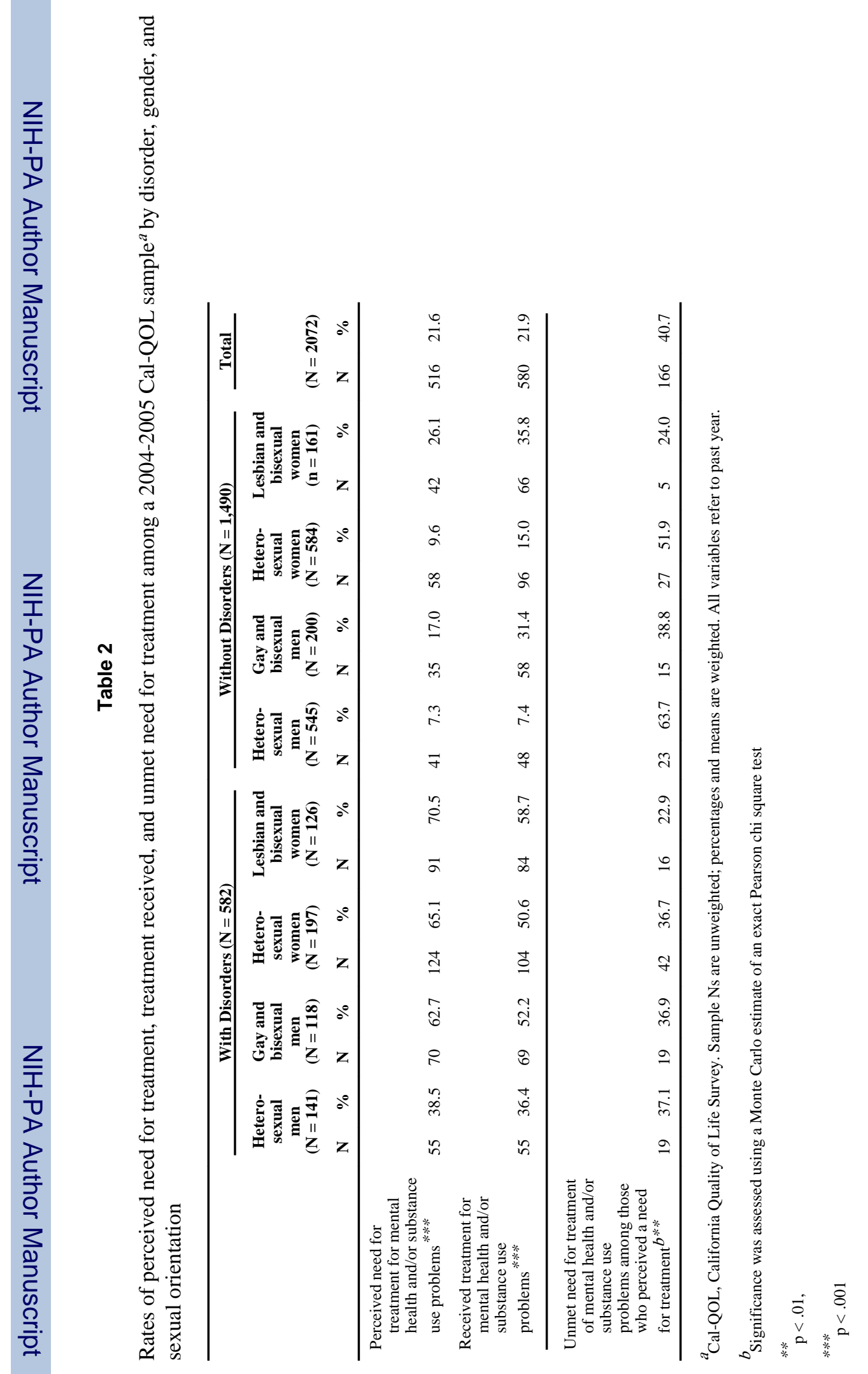




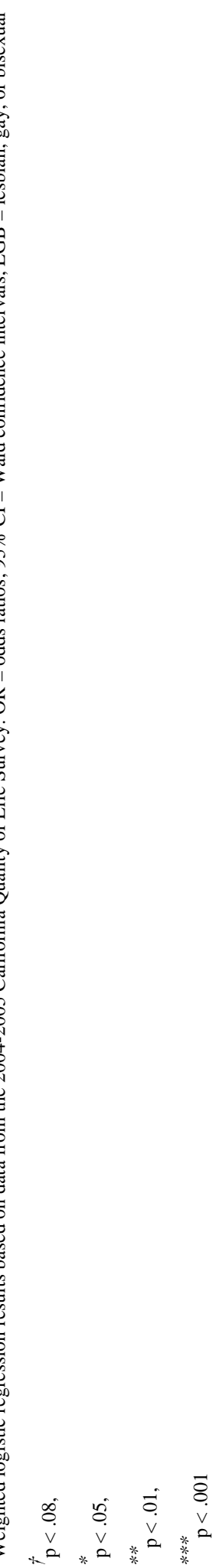

